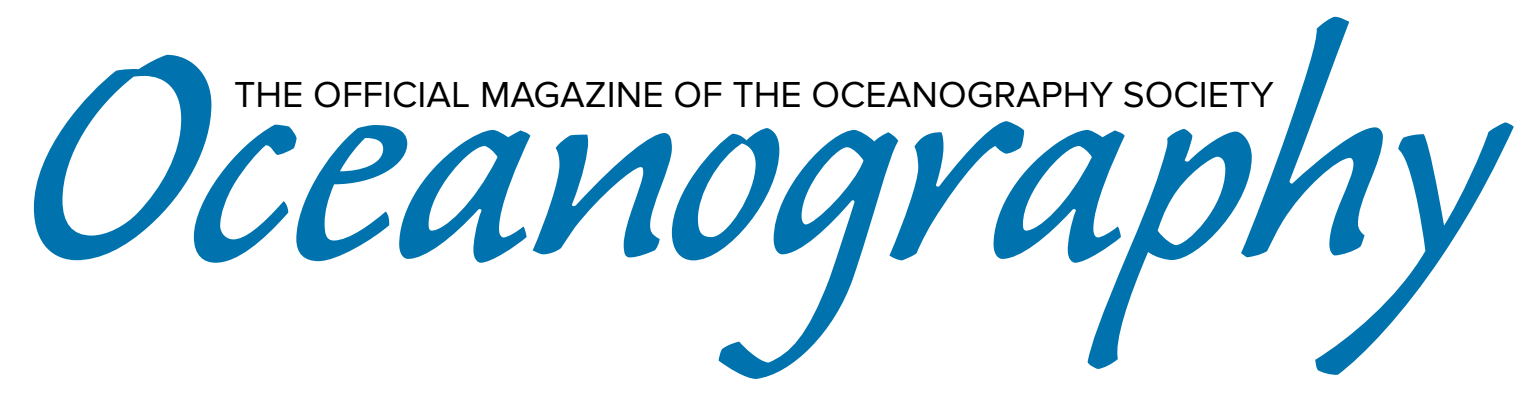

CITATION

Martin, C.I. 2018. On the vorticity of mesoscale ocean currents.

Oceanography 31(3):28-35, https://doi.org/10.5670/oceanog.2018.306.

$\mathrm{DOI}$

https://doi.org/10.5670/oceanog.2018.306

COPYRIGHT

This article has been published in Oceanography, Volume 31, Number 3, a quarterly journal of The Oceanography Society. Copyright 2018 by The Oceanography Society. All rights reserved.

USAGE

Permission is granted to copy this article for use in teaching and research. Republication, systematic reproduction, or collective redistribution of any portion of this article by photocopy machine, reposting, or other means is permitted only with the approval of The Oceanography Society. Send all correspondence to: info@tos.org or The Oceanography Society, 1 Research Court, Suite 450, Rockville, MD 20850, USA. 


\title{
On the Vorticity of Mesoscale Ocean Currents
}

\author{
By Calin Iulian Martin
}

\begin{abstract}
We discuss some aspects of mesoscale ocean flows in which vorticity plays a key role. In addition to wave-current interactions on the continental shelf, where Earth's rotation is negligible, we also look into the interplay of wind forcing and the Coriolis force that drives large-scale ocean eddies.
\end{abstract}

\section{INTRODUCTION}

Vorticity in fluid flows is twice the angular velocity at a point in a fluid and can be considered the local rate of rotation, similar to angular momentum in solids. Imagine that, instantaneously, a tiny part of the fluid continuum becomes solid and the rest of the flow disappears: in this scenario, if the tiny new solid particle is rotating, there is vorticity in the flow. It is important to note that vorticity is the microscopic measure of rotation of a fluid around a point - it is not indicative of global rotation of the fluid (see the discussion in Constantin, 2011a). For discussions of the connections between vorticity and swirling domains of fluid flows, and the challenges of identifying vortices in fluids from features of the velocity field, see Haller (2005) and Haller et al. (2016).

In vector calculus terms, the vorticity vector is simply the curl of the velocity vector. All fluid flows on Earth actually have vorticity simply because of Earth's rotation, with the planetary vorticity vector pointing parallel to Earth's rotation axis. However, in casual observations, we do not consider this type of vorticity because its size is twice the angular rotation rate of Earth, with $\Omega \approx 7.293 \times 10^{-5} \mathrm{rad} \mathrm{s}^{-1}$, so that it is only important for cumulative effects on timescales of the order of a few days and/or spatial scales of the order of tens of kilometers (see Talley et al., 2011). On the other hand, the vorticity of the fluid motion relative to the rigid bed is called the relative vorticity, with the total vorticity of a fluid flow being the sum of the relative vorticity and the planetary vorticity.

In Cartesian coordinates rotating with Earth, with $x, y$, and $z$ directed eastward, northward, and upward, respectively, and with the velocity components designated by $\mathbf{U}=(u, v, w)$, the components of relative vorticity are

$$
\left(\frac{\partial w}{\partial y}-\frac{\partial v}{\partial z}, \frac{\partial u}{\partial z}-\frac{\partial w}{\partial x}, \frac{\partial v}{\partial x}-\frac{\partial u}{\partial y}\right)
$$

while the planetary vorticity vector is given by

$$
2 \Omega(0, \cos \theta, \sin \theta),
$$

where $\theta \in[-\pi / 2, \pi / 2]$ is the angle of latitude. In particular, in the special case of horizontal motion with no $z$ dependence, $\mathbf{U}(x, y)=(u(x, y), v(x, y), 0)$, the relative vorticity has vanishing horizontal components, with vertical component $\zeta(x, y)=\frac{\partial v}{\partial x}-\frac{\partial u}{\partial y}$, and can thus be thought of as a scalar. Also, for $\mathbf{U}(z)=(u(z), 0,0)$, the relative vorticity can be identified with a scalar.

Let us now discuss the importance of the contributions of relative and planetary vorticity to total vorticity in a large-scale ocean flow at mid-latitudes. Typically, the vertical velocity component is about 104 times smaller than the horizontal ones (see Constantin and Johnson, 2017b), and for this reason, we can neglect the contributions of its gradient toward the relative vorticity. Let $U \approx 0.1 \mathrm{~m} \mathrm{~s}^{-1}, H \approx 4 \mathrm{~km}$, and $\mathrm{L} \approx 100 \mathrm{~km}$ be typical values for the horizontal velocity, for the depth, and for the horizontal length scale, respectively, of large-scale ocean flows at mid-latitudes (see Vallis, 2006). A scale analysis of the scalar components of the relative vorticity vector now reveals that the dominant terms in its horizontal components, $\frac{\partial u}{\partial z}$ and $\frac{\partial v}{\partial z}$, scale as $U / H$, whereas the two vertical components, $\frac{\partial u}{\partial y}$ and $\frac{\partial v}{\partial x}$, scale as $U / L$. Therefore, the size of the vertical component of the relative vorticity is $H / L \approx 0.04$ relative to the horizontal components, which means that the relative vorticity vector points mainly in the horizontal direction. The size of the horizontal component of the planetary vorticity is $(2 \Omega \cos \theta) H / U \approx 5$, compared to that of the relative vorticity at $30^{\circ}$ latitude. On the other hand, considering the vertical components of the two vorticity vectors, the ratio of the relative and planetary contributions to the total vorticity scales as $U /(2 L \sin \theta) \approx 10^{-2}$. These considerations 
show that for large-scale ocean flows at mid-latitudes, the relative vorticity is typically small compared to the planetary vorticity. However, there are exceptions to this rule: eddies are encountered in all regions of the global ocean. Following Talley et al. (2011), by "eddies" we mean features with horizontal scales in excess of several kilometers, up to about $200 \mathrm{~km}$, that are departures from the mean properties described above. At these mesoscales, the issue of surface waves might also present intriguing facets that are simply irrelevant for large-scale ocean flows, and for which the effects of Earth's rotation are minute. In this paper we explore these two directions of investigation from the vantage point of vorticity, within the setting of steady flows-although there is definitely some temporal variability in the wind field, nevertheless, a persistent average pattern is evident (e.g., https:// www.opc.ncep.noaa.gov/gridded_wind_vectors.php), and this justifies the use of a steady-flow framework for mesoscale ocean circulation. Note also that while the relative vorticity of ocean flows is mainly wind generated, the way in which other factors come into play in the global ocean circulation (e.g., the Coriolis force) is often quite subtle-for example, the North Equatorial Countercurrent flows upwind in the Pacific Ocean.

\section{WAVE-CURRENT INTERACTIONS WITH NEGLIGIBLE CORIOLIS EFFECTS}

The continental shelf is an underwater landmass, with average depth about $50 \mathrm{~m}$, that extends seaward from the shore with a typical gradient of $1 / 500$, while at its outer limit-the shelf break-the gradient increases precipitously to about $1 / 20$ to form the continental slope down to the sea bottom at several kilometers depth. The continental shelf of Eastern Siberia is the widest continental shelf, extending more than $1,000 \mathrm{~km}$ offshore, along a continental coastline that exceeds $3,000 \mathrm{~km}$ (see Figure 1). It is covered by ice for most of the year, except in the summer, when 2-3 $\mathrm{m}$ high waves with wavelengths of the order of $60-90 \mathrm{~m}$ propagate at the surface of a flat bed of mean depth $45 \mathrm{~m}$ (see Münchow et al., 1999).

The most important currents on continental shelves are tidal and wind-generated currents. Tidal currents flow toward the shore (flood state) during part of the tidal cycle, clustered around $24 \mathrm{~h}$ and $12 \mathrm{~h}$ bands (diurnal and semidiurnal, respectively), and seaward (ebb state) during the remainder of the tidal cycle, with slack water periods (almost no horizontal movement) in the transition period. The unsteadiness of tidal currents is relevant for timescales of several hours, while for times of the order of one hour, tidal currents are nearly uniform with depth (see Peregrine and Jonsson, 1983). Although tide-generating forces are all-pervasive in the ocean, they do not influence the mean large-scale ocean circulation because they represent periodic motions that do not contribute to the balance of forces for the steady state. Over timescales of the order of one hour, the tidal current is uniform with depth and propagates in one direction as an irrotational flow (with zero vorticity), with free-surface waves of insignificant wave height (of a few centimeters), as depicted in Figure 2. Tidal current speeds on the East Siberian continental shelf are of the order of $0.05 \mathrm{~m} \mathrm{~s}^{-1}$ while the speed of the surface wind-drift current in this region is about $0.5 \mathrm{~m} \mathrm{~s}^{-1}$ (and rarely exceeds $1.5 \mathrm{~m} \mathrm{~s}^{-1}$; see Münchow et al., 1999). The challenge is therefore to investigate the wind-driven flow. Over scales of the order of hundreds of meters (for which the effects of Earth's rotation are insignificant), when wind blows over water, it accelerates the surface in the wind direction, and the velocity profile of the wind-drift current develops in the near-surface region. Systematic data collection provides guidance for the velocities of wind-generated currents as functions of depth, with the horizontal fluid velocity profile of the pure current state over the flat bed $z=-d$, in the absence of waveinduced vertical velocities, given by

$$
u_{0}(z)=\left\{\begin{array}{l}
s\left(1+\frac{z}{d_{0}}\right) \text { for }-d_{0} \leq z \leq 0 \\
0 \text { for }-d \leq z \leq-d_{0}
\end{array}\right.
$$

where $d_{0}>0$ is the reference depth of $50 \mathrm{~m}$, and the magnitude $s$ of the surface wind-drift (at $z=0$ ) corresponds to about $2 \%-7 \%$ of the wind velocity measured at $10 \mathrm{~m}$ above sea level (see Ewing,

\section{East Siberian Arctic Shelf}

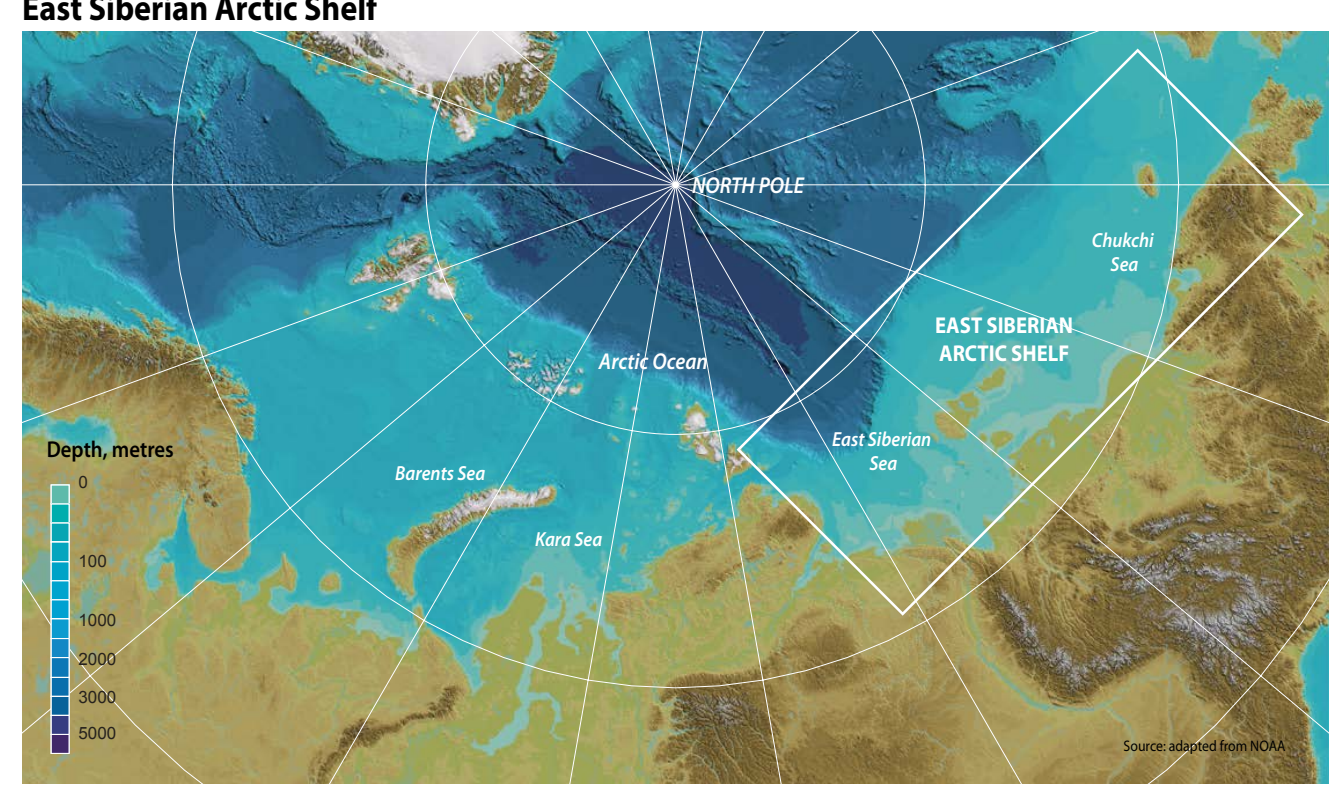

FIGURE 1. Arctic Ocean seafloor map with key bathymetric features noted and the area of the East Siberian continental shelf highlighted. (OGRIDArendal 
1990). The flow pattern of the eastward wind-drift current on the Eastern Siberian continental shelf is depicted in Figure 3.

As noted in the introduction, the pure-current flow (1) has non-zero constant vorticity $s / d_{0}$. In this case, the twodimensional nature of the flow permits us to regard the vorticity as a scalar, and in the presence of waves, this parallel flow is distorted by the wave-induced vertical motion in wavecurrent interaction (see Figure 4 for an illustration of traveling waves, flows whose velocity field and free surface exhibit an $(x, t)$-dependence in the form $(x-c t)$, where $x$ is the direction of propagation, $t$ is time, and $c$ is the constant wave speed. The wave action distorts the straight horizontal streamline-pattern observed in the pure-current state (in the absence of waves, as depicted in Figures 2 and 3) into sinusoidal curves with an oscillation amplitude that typically decays with depth, so that the oscillation subsides on the flat bed (with the motion along the bed in the direction of wave propagation).

Note that the flow pattern for a fixed observer differs markedly from that depicted in Figure 4 (in which the observer follows the wave), even in the case when the current is uniform and the flow is irrotational (zero vorticity): every particle experiences a back-and-forth motion in the horizontal direction, with a forward drift. In the case of particles above the flat bed, the motion also has an oscillatory character in the vertical direction, so that the particle describes a forward loop (see the discussion in Constantin (2006, 2011a) and Constantin and Strauss (2010) for theoretical considerations, Clamond (2012) for numerical simulations, and Umeyama (2012) for experimental evidence). Study of particle paths beneath a traveling water wave in a flow without an underlying current indicates that nonlinear effects are essential. Neglecting them by using a linear approximation leads to erroneous theoretical conclusions that in a fixed reference frame the particle paths are closed. For example, in water with a flat horizontal bed, all particles move on ellipses that have the same distance between their foci, but the lengths of their axes

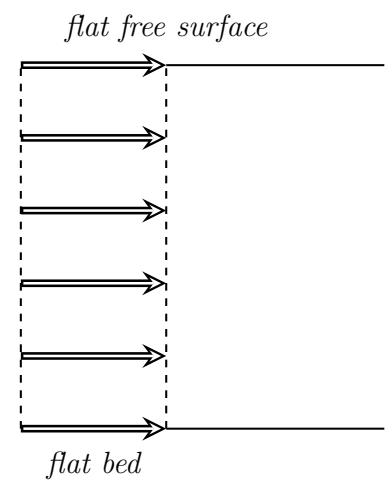

FIGURE 2. Uniform twodimensional current as a model for a free-surface irrotational flow in the absence of waves. Tidal currents present such features.

FIGURE 3. Sheared two-dimensional current without flow reversal as a model for a free-surface flow with constant non-zero vorticity in the absence of waves. Wind-generated small-scale currents on the conti- decrease with depth, so that at the bottom the ellipses degenerate into straight lines; moreover, each particle appears to describe its ellipse in a wave period, and they are all in the same phase, whereas in reality they perform a backward-forward motion (a loop pattern) with a forward drift (see Milne-Thomson, 1960; Constantin, 2011a).

It is quite difficult to gather information about the underlying current from knowledge of the free surface, with the pure wave state (no underlying current, which could happen if waves generated by a distant storm enter an area of still water) being quite similar to that of a wave-current interaction with a uniform underlying current, or even with a wind-drift current that moves in the direction of the waves. Some counterintuitive phenomena occur, for example, the fact that in the absence of adverse currents, a free-surface profile that is monotone between successive crests and troughs has to be symmetric even if an underlying sheared current is present (see Constantin and Escher, 2004; Constantin et al., 2007). This means that the shear of the underlying current is not easily recognizable in the surface wave pattern. Note that though surface waves can be generated by local short-duration winds and can propagate over large distances, the underlying current is due to long-duration, persistent wind action. Thus, in a wave-current interaction, the direction of wave propagation and of the underlying current may be different. In particular, adverse currents propagating in the direction opposite to that in which the surface waves move may be encountered. We distinguish between favorable currents, which are sheared in the same direction as that of the wave propagation (downstream waves), and adverse currents, sheared opposite to the direction of wave propagation (upstream waves). Field data, laboratory experiments, and numerical simulations indicate that an adverse wind-drift current will shorten the wavelength, and thereby increase both wave height and wave steepness, even to the extent that bulbous waves with overhanging wave profiles might appear (see the discussions in daSilva and

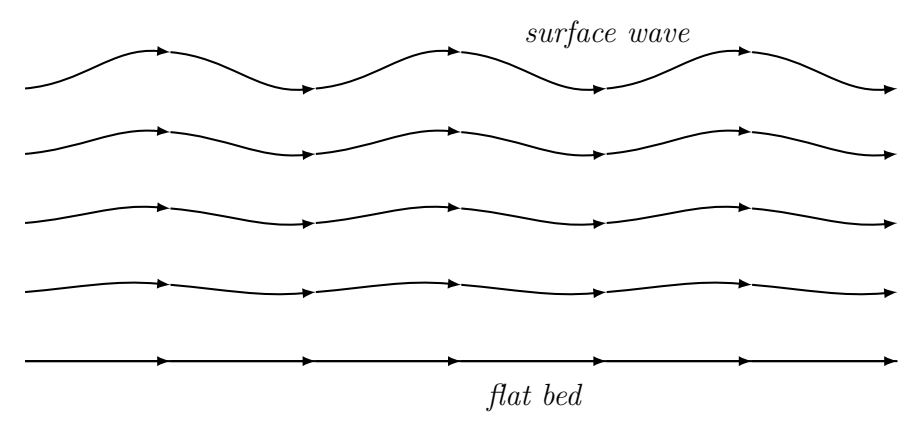
nental shelf are of this type.
FIGURE 4. Streamlines of a wave-current interaction between a surface traveling wave and an underlying current of constant vorticity without flow reversal, in the frame of reference moving at a constant wave speed (in which the flow appears steady). 
Peregrine, 1988; Swan et al., 2001; Constantin et al., 2016). In contrast, for waves propagating in the direction of the current, the wavelength becomes longer and flattens the wave so that its slopes are less steep (see Thomas, 1990; Ko and Strauss, 2008; Constantin et al., 2015).

The presence of an adverse current can lead to the appearance of critical levels where the background current equals the wave's phase velocity (so that the speed vanishes in the reference frame moving at the wave speed) and flow-reversal occurs (as depicted at mid-depth in Figure 5). Critical levels are of great importance in wave-current interactions because they considerably alter the flow pattern, with drastic changes possible due to the action of waves (e.g., the flow reversal of the subsurface current field in the equatorial Pacific and the occurrence of slow, long, zonal, internal waves lead to the formation of critical levels; see the discussions in Smyth et al., 2011, Constantin, and Johnson, 2015, and Liu et al., 2016). Stagnation points trigger these phenomena (stagnation points occur when the horizontal fluid velocity equals the wave speed and the vertical fluid velocity vanishes, so that in a frame moving at the wave speed these points are stagnant; see Figure 6 and studies of the flow beneath a surface wave with vorticity: Wahlen, 2009; Constantin and Varvaruca, 2011; Constantin et al., 2016; Ribeiro et al., 2017). While in Earth's ocean the currents are nowhere near fast enough to match the speed of a surface gravity wave (among the fastest currents are those between the Scottish mainland and the Orkney Islands in Pentland Firth, with speeds up to $5.5 \mathrm{~m} \mathrm{~s}^{-1}$; see Goddijn-Murphy et al., 2013), internal waves can develop critical levels (e.g., in the equatorial Pacific, as pointed out above). Critical levels are absent in irrotational flows, so that non-zero vorticity (associated with shear flows) is essential for the occurrence of such flow patterns. Moreover, note that in the case of the East Siberian continental shelf, the wind-drift current reaches to the bottom of the shelf because the shelf is a relatively shallow region. In deeper regions, the wind-drift current may be confined to a near-surface layer beneath which the flow is irrotational. The simplest description of such a scenario, from the point of view of vorticity, would be a discontinuous vorticity distribution, in the sense that the fluid consists of two layers of constant but different vorticities. For investigations of this phenomenon, see Henry (2013), Martin (2014, 2015, 2017a), and Martin and Matioc (2016).

The setting for the considerations regarding wave-current interactions described above is that of two-dimensional inviscid gravity water flows with constant vorticity. Inviscid theory is appropriate for the study of water waves that are not near breaking because the most significant effects of viscosity in the open sea are wave-amplitude reduction and diffusion of deeper motions over time and length scales that are far larger than those of the dynamical surface processes (see daSilva and Peregrine, 1988). Also, the choice of constant vorticity is justified because for long waves propagating at the water's surface over a nearly flat bed (with wavelengths that exceed the mean water depth), the existence of non-zero mean vorticity is more important than its specific distribution (see the discussion in daSilva and Peregrine, 1988). Using the governing equations (incompressible Euler equations), it can be shown that in a two-dimensional flow, the vorticity of each individual water particle is constant as the particle moves about (see the discussion in Constantin, 2011a). In particular, if the vorticity of a two-dimensional flow is constant at an instant, it will remain so in its subsequent evolution. In contrast to this, there is a lack of conservation of vorticity in three-dimensional flows, and this feature proves to be a major obstacle to the understanding of crucial properties of the flow. Nevertheless, the governing equations indicate that in three-dimensional flows a particle that has no vorticity never acquires it (see Constantin, 2011a). In general, part of the water may be moving irrotationally and other parts rotationally. As time passes, the irrotational part may occupy different regions of space: the fact that the vorticity vanishes is a property of those parts of the fluid that are moving irrotationally and not of the

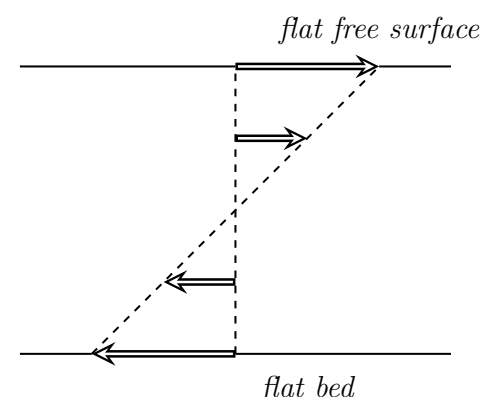

FIGURE 5. Sheared two-dimensional current with flow reversal as a model for a free-surface flow with constant non-zero vorticity in the absence of waves. Wind-generated small-scale currents (for which the effect of Earth's rotation is not relevant) interacting with a uniform countercurrent (e.g., a tidal current) are of this type.

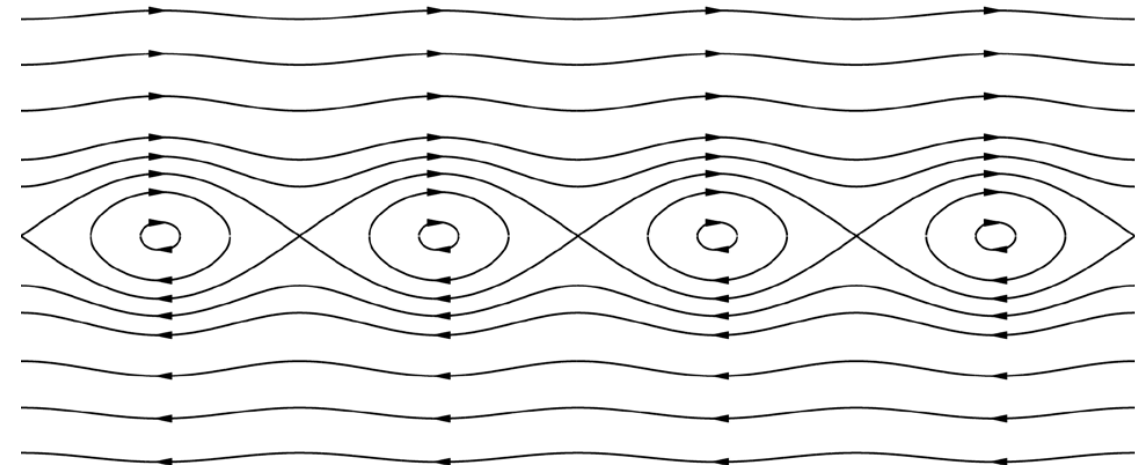

FIGURE 6. Kelvin's cat's eye streamline pattern, as viewed by an observer moving with the wave speed, is typical for traveling surface waves interacting with an underlying current of constant vorticity with flow reversal. The wave action distorts the pattern depicted in Figure 4 in the absence of a countercurrent, with the most striking changes occurring near the level of the horizontal critical layer consisting of stagnation points that mark flow reversal. Only the points of the critical layer that lie beneath the crest or the trough remain stagnation points, as they are surrounded by closed streamlines that delimit an upper region of positive horizontal fluid velocity from a lower fluid region in which the flow direction is reversed. 
regions of space they may occupy at some instant. Therefore, if a fluid flow has regions that are irrotational and others where non-zero vorticity is present, the spatial region originally occupied by fluid in irrotational motion may pick up vorticity at later times, so, in general, the irrotational region will move around with the flow.

The localized nature of wave-current interactions makes the effects of Earth's rotation (and of its sphericity) negligible. We did not address the way in which energy is transferred from the wind to the water, taking the view that we only study the propagation of waves on currents once both are generated. We also note that the effects of surface tension related to the appearance of capillary or capillary gravity water waves are on much smaller scales, of the order of millimeters and centimeters, respectively, and for this reason we did not take them into account. While it might look somewhat restrictive, two-dimensionality (in the form of a preferred direction of propagation and variations that essentially occur only in this horizontal direction and in that of the vertical, so that to describe these flows it suffices to consider a cross section in the direction of wave propagation since the motion is identical in any plane parallel to it), it is actually of great physical relevance for wave-current interactions. Indeed, the primary sources of currents are long-duration winds. Systematic studies of the velocity profiles of wind-generated currents in shallow-water regions with a nearly flat bed, such as the continental shelves, show that they are accurately described as flows with constant vorticity (Ewing, 1990). Also, gravity water

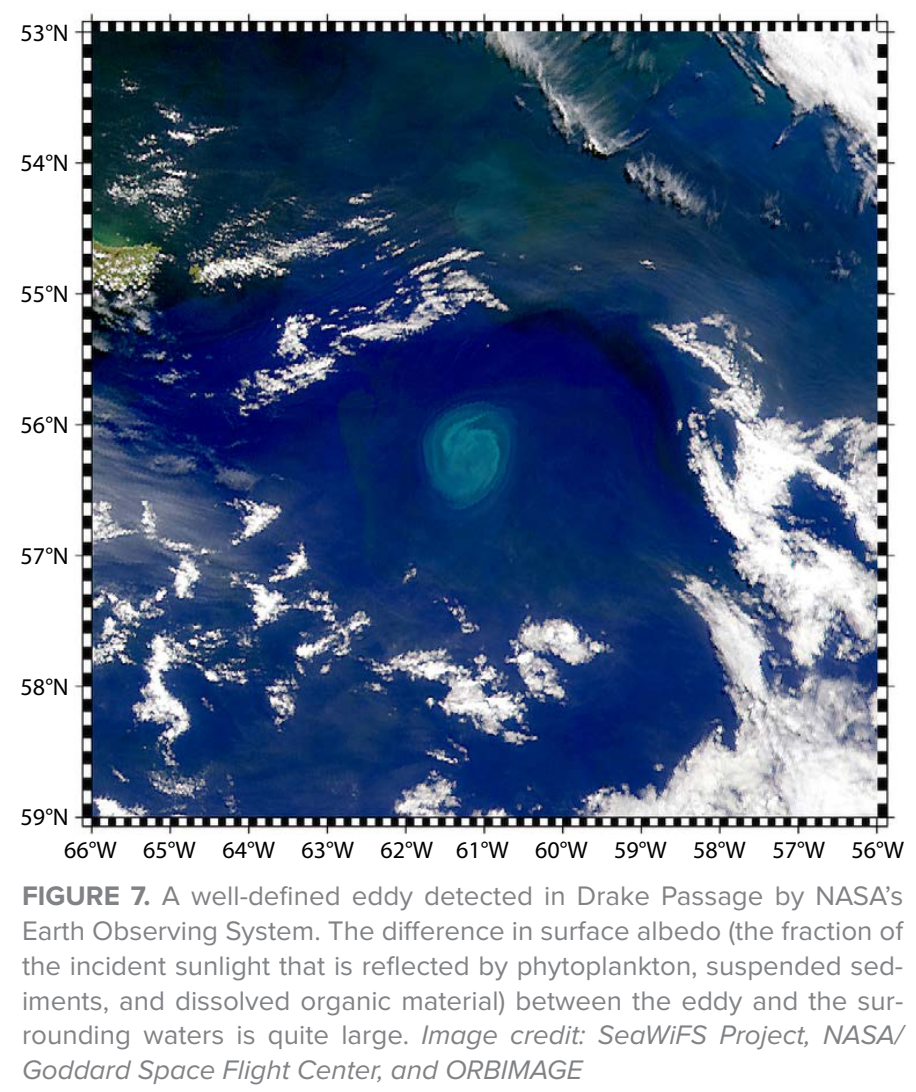

flows of constant vorticity are inherently two-dimensional, with the vorticity determining the direction of wave propagation (see the discussions in Constantin, 2011b; Wahlen, 2014; Martin, 2017c). The main conclusion of these considerations is that vorticity is a hallmark of intricate ocean flow patterns, even without accounting for the effects of Earth's rotation and sphericity. The effects of Earth's rotation and also those of its sphericity (for large-scale ocean flows) can only enrich the dynamics, adding to the intricacy of the investigation, but also bringing about new phenomena.

\section{LARGE-SCALE EDDIES}

To illustrate how Earth's rotation induces deflections in the near-surface ocean flow as a result of wind action, we offer a case study of the eddy depicted in Figure 7, located in Drake Passage in a region where there is a nearly flat bed (see Figure 8).

Drake Passage is an oceanic gateway approximately $850 \mathrm{~km}$ wide. Located between South America and Antarctica, it connects the Pacific and the Atlantic Oceans and constricts the Antarctic Circumpolar Current (ACC) to a narrow geographical region. For the last 20 years, Drake Passage has been the most comprehensively observed part of the Southern Ocean. Geological studies of oceanic sedimentary rock in the passage show that it was closed about 41 million years ago, and before it opened, the Atlantic and Pacific Oceans were entirely separate, with Antarctica being much warmer (in particular, having no ice cap). The joining of the two great oceans initiated the ACC and resulted in a significant cooling of Antarctica by about $3^{\circ} \mathrm{C}$ (see Toggweiler and Bjornsson, 2000). Drake Passage, whose average depth is about $4 \mathrm{~km}$, is the only means of exchange of deep waters between the Pacific and the Atlantic Oceans because the depth of the Bering Strait between Alaska and Siberia varies between only 30 and $50 \mathrm{~m}$. Moreover, the flow through this narrow and shallow strait (it is $82 \mathrm{~km}$ wide at its narrowest point) is much less than that through Drake Passage.

The ACC is the most important current in the Southern Ocean, and the only current that flows completely around Earth, encircling the continent of Antarctica and flowing eastward through the southern parts of the Atlantic, Indian, and Pacific Oceans. The ACC is often referred to as the "West Wind Drift" because it is wind-generated, and the prevailing westerly wind in this ocean region is eastward. In contrast to most wind-generated currents, which are typically confined to the upper $200 \mathrm{~m}$ of the ocean, the ACC reaches great depths, with speeds of $0.05 \mathrm{~m} \mathrm{~s}^{-1}$ at about $2 \mathrm{~km}$ depth, while the surface speed attains $0.15 \mathrm{~m} \mathrm{~s}^{-1}$ (see the discussion in Constantin and Johnson, 2016b). While significant surface waves interact with the ACC-monster waves more than $35 \mathrm{~m}$ high are typically observed near the southern tip of Africa (see Walton, 2013) - and many aspects of wavecurrent interactions are of current interest (see Constantin and Monismith, 2017), for studying the eddy in Drake Passage, only the current aspect of the ACC is of significance, given that the 
relevant scale is of the order of $10 \mathrm{~km}$ (mesoscale). ACC flow has non-zero vorticity, but wind stress also plays a role in driving the eddy. As strong winds blow across the Southern Ocean, the water moves as a result of its frictional drag on the surface. If we ignore Earth's rotation, as we did in the earlier section on WaveCurrent Interactions, then frictional coupling between the moving air and the ocean surface would push a thin layer of water in the same direction as the wind. The surface layer in turn would drag the layer beneath it, putting it into motion, and this interaction would propagate downward through successive ocean layers, like cards in a deck, each lower layer moving in the wind direction at a slower speed than the layer above it (thus generating the sheared current profile depicted in Figure 3). This type of behavior occurs not only in small-scale flows (like those discussed in the section above on Wave-Current Interactions) but also in large-scale equatorial ocean flows, because in both cases the Coriolis effect is negligible-at the equator the Coriolis effect is zero. We refer the reader to Constantin, (2012), Constantin and Johnson (2015, 2016a, 2017a), Henry (2016), Martin (2017b, 2017c), and Ionescu-Kruse and Martin (2018) for various aspects of equatorial flows, and discuss here some general features of mid-latitude wind-drift currents that are applicable to the Southern Ocean. At mid-latitudes the effect of Earth's rotation is to deflect, to depths of about 100-150 m, each water layer with respect to the one above it, while the motion of the surface water is deflected relative to that of the wind. This behavior is known as the Ekman spiral, named for the Swedish physicist Vagn Walfrid Ekman (1874-1954) who first investigated it mathematically (Ekman, 1905). Ekman was prompted to study this phenomenon by field observations made by the Norwegian explorer Fridtjof Nansen (1861-1930), who attempted in 1893 to reach the North Pole by allowing his wooden ship, Fram, to freeze into the ice pack (where it remained locked for about 35 months) in the hope to drift with the ice and thereby reach the North Pole. While Nansen only came within $394 \mathrm{~km}$ of the North Pole, he made a number of important observations. In particular, he noticed that the direction of ice and ship movement was consistently to the right of the prevailing wind direction. Ekman's reasoning was that, by neglecting the vertical velocity component $(w)$ and retaining only the horizontal velocity components $(u, v)$, at leading order in the mesoscale regime in which Coriolis effects matter and within the layer in which friction plays a role in the balance of forces (now called Ekman layer), the linear approximation of the governing equations reduces to the system

$$
\begin{aligned}
-f v & =-\frac{1}{\rho} \frac{\partial P}{\partial x}+\nu \frac{\partial^{2} u}{\partial z^{2}} \\
f u & =-\frac{1}{\rho} \frac{\partial P}{\partial y}+\nu \frac{\partial^{2} v}{\partial z^{2}} \\
0 & =-g-\frac{1}{\rho} \frac{\partial P}{\partial z} \\
0 & =\frac{\partial u}{\partial x}+\frac{\partial v}{\partial y}
\end{aligned}
$$

where $x$ is the zonal direction (west-to-east), $y$ is the meridional direction (northwards), and $z$ is that of the local vertical, while $\rho$ is the (constant) density, $v$ is the (constant) eddy viscosity, $g$ is the gravitational constant of acceleration (whose value is taken to be $9.81 \mathrm{~m} \mathrm{~s}^{-2}$ ), $P$ is the pressure variable, and

$$
\mathrm{f}=2 \Omega \sin \theta_{0}
$$

is the Coriolis parameter for the relevant latitude $\theta_{0}$ (see Vallis, 2006). The appropriate boundary conditions are

$$
\begin{gathered}
u_{z}=\tau_{1} \text { and } v_{z}=\tau_{2} \text { on } z=0, \\
P=P_{a t m} \text { on } z=0,
\end{gathered}
$$

$(u, v)$ decays beneath the surface,

where $P_{a t m}$ is the (constant) atmospheric pressure while $\tau_{1}$ and $\tau_{2}$ are the components of the wind stress on the free surface $z=0$. The system (2)-(5) is derived in the $f$-plane approximation of the Navier-Stokes equations on a rotating sphere, which permits locally flat-space geometry so that, in particular, the free surface is regarded as a part of the tangent plane, which is identified with $z=0$ (see the discussion in Vallis, 2006). From (4) and (8) we infer that the pressure is hydrostatic throughout the Ekman layer, being given by $P(x, y, z)=P_{a t m}-\rho g z$,

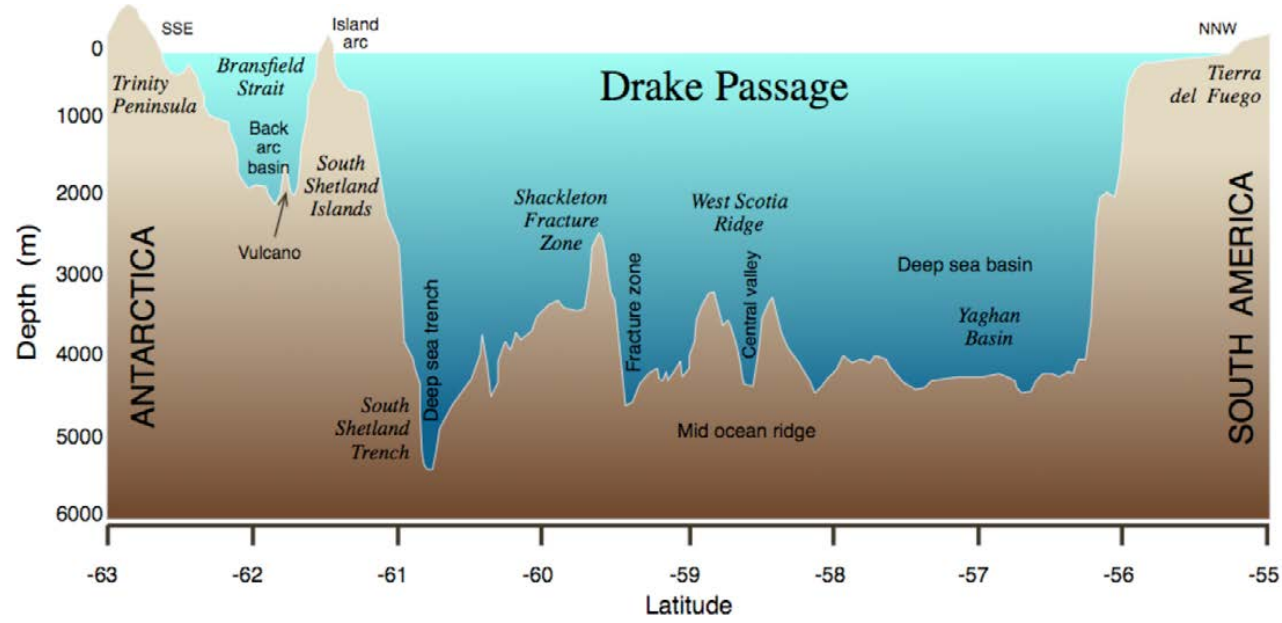

FIGURE 8. Bathymetry of Drake Passage. The ocean floor is mostly flat except for a series of submerged ridges that extend up from the bed. In particular, a flat bed is typical for the region between the circles of latitude $56^{\circ} \mathrm{S}$ and $58^{\circ} \mathrm{S}$, in which the eddy shown in Figure 7 is located. Image credit: Hannes Grobe, Alfred Wegener Institute for Polar and Marine Research 
so that the system (2)-(5) simplifies to

$$
\begin{gathered}
-f v=\nu \frac{\partial^{2} u}{\partial z^{2}}, \\
f u=\nu \frac{\partial^{2} v}{\partial z^{2}}, \\
0=\frac{\partial u}{\partial x}+\frac{\partial v}{\partial y},
\end{gathered}
$$

with boundary conditions shown in (7) and (9). Introducing the complex-valued function

$$
F=u+i v
$$

and setting

$$
d=\sqrt{\frac{|f|}{2 \nu}}
$$

we get (see Vallis, 2006)

$$
F=A(x, y) \exp \{-[1+i \operatorname{sgn}(\theta)] d z\},
$$

for some complex function $A(x, y)$. Note that (13) yields

$$
\frac{F_{z}}{F}=-[1+i \operatorname{sgn}(\theta)] d \quad \text { on } \quad z=0 .
$$

The fact that the argument of the complex number on the right side of the above relation is $\left\{-\frac{\pi}{4} \operatorname{sgn}(\theta)\right\}$ means that the windgenerated surface current is at an angle of $\frac{\pi}{4}$ with respect to the wind stress at the surface, deflected to the right in the Northern Hemisphere and to the left in the Southern Hemisphere. This solution is basically the classical Ekman spiral: from (13) we see that the horizontal velocity field rotates and decays with depth in non-equatorial regions, like that of the gyre in Drake Passage.

We can now interpret the classical Ekman solution in the near-surface Ekman layer in terms of the relative vorticity of the flow. Taking the derivative of (10) with respect to the $y$-variable and the derivative of (11) with respect to the $x$-variable and subtracting the resulting equations leads to the vorticity equation

$$
\frac{\partial^{2} \gamma}{\partial z^{2}}=0
$$

Based on (5); we denote by

$$
\gamma=\frac{\partial v}{\partial x}-\frac{\partial u}{\partial y}
$$

the relative vorticity of the flow. Note that, by neglecting the vertical fluid velocity, the flow is merely a horizontal flow and thus the vorticity can be thought of as a scalar (see the discussion in the introduction). From (14) we infer that

$$
\gamma=\gamma_{1} z+\gamma_{0}
$$

for some real constants $\gamma_{0}$ and $\gamma_{1}$. Due to (9), the relative vorticity should become negligible at great depths so that the only option is that $\gamma_{1}=\gamma_{0}=0$ in (16), and therefore the relative vorticity is zero. Alternatively, from the explicit form (13) of the horizontal velocity field in Ekman's classical solution, we see that $\gamma=0$. Indeed, using the polar decomposition

$$
A(x, y)=a(x, y) \exp [i \beta(x, y)]
$$

with $a \geq 0$ and $\beta \in[0,2 \pi]$, from (13) we get

$$
\left\{\begin{array}{l}
u=a \exp (-d z) \cos [\beta-d z \operatorname{sgn}(\theta)] \\
v=a \exp (-d z) \sin [\beta-d z \operatorname{sgn}(\theta)] .
\end{array}\right.
$$

Now (5) holds if and only if

$$
\left\{\begin{array}{l}
a_{x}+a \beta_{y}=0 \\
a_{y}-a \beta_{x}=0
\end{array}\right.
$$

in particular, for $a>0$ the functions $\beta$ and $\ln a$ are harmonic conjugated, with the complex-valued function $a \exp (-i \beta)$ analytic in the complex variable $x+i y$. A direct computation confirms now from (17) to (18) that the horizontal flow is irrotational, that is, $\gamma=v_{x}-u_{y}=0$.

This situation is clearly unsatisfactory and calls for further development of Ekman's ideas. A possibility that was explored within the oceanographic research literature was to go beyond the $f$-plane approximation using the $\beta$-plane approximation that retains the variation of the Coriolis parameter with latitude. In this case, rather than $f=2 \Omega \sin \theta_{0}$, we use the adjustment

$$
f=2 \Omega\left(\sin \theta_{0}+y \cos \theta_{0}\right),
$$

which captures to first order a variation with latitude. The approach presented above can be implemented, leading again to a spiraling horizontal current, deflected at the surface by $\pm \frac{\pi}{4}$ with respect to the wind direction but in this case $d$ is $y$-dependent in the solution (13), rather than constant. The system (18) associated with the solution given by (17) is replaced by

$$
\left\{\begin{array}{l}
a_{x}+a \beta_{y}-z a d_{y} \operatorname{sgn}(\theta)=0, \\
a_{y}-a \beta_{x}-z a d_{y}=0 .
\end{array}\right.
$$

Unless $a \equiv 0$, the system (19) is incompatible, which illustrates a general issue related to the $\beta$-plane approximation (see the discussion in Dellar, 2011). The remaining option is to dispense with approximations and to use rotating spherical coordinates, a research direction of current interest (Constantin and Johnson, 2018).

\section{CONCLUSION}

We showed that for wave-current interactions on the continental shelf, vorticity is a measure of the complexity of the flow pattern and offers insight into important aspects. However, the role of vorticity in mesoscale ocean flows, which requires accounting for the effects of Earth's rotation, remains to be explored. In particular, the vorticity effect on the shape of the surface waves is of great interest. Our considerations show also the importance of depth-dependent eddy viscosities in generating Ekman-type flows with non-zero vorticity, given that the presence of vorticity in the near surface ocean layers is experimentally well established. @ 


\section{REFERENCES}

Clamond, D. 2012. Note on the velocity and related fields of steady irrotational two-dimensional surface gravity waves. Philosophical Transactions of the Royal Society A 370:1,572-1,586, https://doi.org/10.1098/rsta.2011.0470.

Constantin, A. 2006. The trajectories of particles in Stokes waves. Inventiones Mathematicae 166:523-535, https://doi.org/10.1007/s00222-006-0002-5.

Constantin, A. 2011a. Nonlinear water waves with applications to wave-current interactions and tsunamis. CBMS-NSF Regional Conference Series in Applied Mathematics, SIAM, Philadelphia, https://doi.org/10.1137/1.9781611971873.

Constantin, A. 2011b. Two-dimensionality of gravity water flows of constant nonzero vorticity beneath a surface wave train. European Journal of Mechanics B/Fluids 30:12-16, https://doi.org/10.1016/j.euromechflu.2010.09.008.

Constantin, A. 2012. An exact solution for equatorially trapped waves. Journal of Geophysical Research 117, C05029, https://doi.org/10.1029/2012JC007879.

Constantin, A., M. Ehrnström, and E. Wahlen. 2007. Symmetry of steady periodic gravity water waves with vorticity. Duke Mathematical Journal 140:591-603.

Constantin, A., and J. Escher. 2004. Symmetry of steady periodic surface water waves with vorticity. Journal of Fluid Mechanics 498:171-181, https://doi.org/10.1017/S0022112003006773.

Constantin, A., and R.S. Johnson. 2015. The dynamics of waves interacting with the Equatorial Undercurrent. Geophysical \& Astrophysical Fluid Dynamics 109:311-358, https://doi.org/10.1080/03091929.2015.1066785.

Constantin, A., and R.S. Johnson. 2016a. An exact, steady, purely azimuthal equatorial flow with a free surface. Journal of Physical Oceanography 46:1,935-1,945, https://doi.org/10.1175/JPO-D-15-0205.1.

Constantin, A., and R.S. Johnson. 2016b. An exact, steady, purely azimuthal flow as a model for the Antarctic Circumpolar Current. Journal of Physical Oceanography 46:3,585-3,594, https://doi.org/10.1175/JPO-D-16-0121.1.

Constantin, A., and R.S. Johnson. 2017a. A nonlinear, three-dimensional model for ocean flows, motivated by some observations of the Pacific equatorial undercurrent and thermocline. Physics of Fluids 29, 056604, https://doi.org/ 10.1063/1.4984001.

Constantin, A., and R.S. Johnson. 2017b. Large gyres as a shallow-water asymptotic solution of Euler's equation in spherical coordinates. Proceedings of the Royal Society A 473, https://doi.org/10.1098/rspa.2017.0063.

Constantin, A., and R.S. Johnson. 2018. Steady large-scale ocean flows in spherical coordinates. Oceanography 31(3):42-50, https://doi.org/10.5670/ oceanog.2018.308.

Constantin, A., K. Kalimeris, and O. Scherzer. 2015. A penalization method for calculating the flow beneath traveling water waves of large amplitude. SIAM Journal of Applied Mathematics 75:1,513-1,535, https://doi.org/10.1137/14096966X.

Constantin, A., and S.G. Monismith. 2017. Gerstner waves in the presence of mean currents and rotation. Journal of Fluid Mechanics 820:511-528, https://oi.org/ 10.1017/jfm.2017.223.

Constantin, A., and W. Strauss. 2010. Pressure beneath a Stokes wave. Communications on Pure and Applied Mathematics 63:533-557, https://doi.org/ 10.1002/cpa.20299.

Constantin, A., W. Strauss, and E. Varvaruca. 2016. Global bifurcation of steady gravity water waves with critical layers. Acta Mathematica 217:195-262, https://doi.org/10.1007/s11511-017-0144-x.

Constantin, A., and E. Varvaruca. 2011. Steady periodic water waves with constant vorticity: Regularity and local bifurcation. Archive for Rational Mechanics and Analysis 199:33-67, https://doi.org/10.1007/s00205-010-0314-x.

daSilva, A.F.T., and D.H. Peregrine. 1988. Steep, steady surface waves on water of finite depth with constant vorticity. Journal of Fluid Mechanics 195:281-302 https://doi.org/10.1017/S0022112088002423.

Dellar, P.J. 2011. Variations on a beta-plane: Derivation of non-traditional betaplane equations from Hamilton's principle on a sphere. Journal of Fluid Mechanics 674:174-195, https://doi.org/10.1017/S0022112010006464.

Ekman, V.W. 1905. On the influence of the Earth's rotation on ocean currents. Arkiv för matematik, astronomi och fysik 2:1-52.

Ewing, J.A. 1990. Wind, wave and current data for the design of ships and offshore structures. Marine Structures 3:421-459, https://doi.org/10.1016/ 0951-8339(90)90001-8.

Goddijn-Murphy, L., D.K. Woolf, and M.C. Easton. 2013. Current patterns in the Inner Sound (Pentland Firth) from underway ADCP data. Journal of Atmospheric and Oceanic Technology 30:96-111, https://doi.org/10.1175/JTECH-D-11-00223.1.

Haller, G. 2005. An objective definition of a vortex. Journal of Fluid Mechanics 525:1-26, https://doi.org/10.1017/S0022112004002526.

Haller, G., A Hadjighasem, M. Farazmand, and F. Huhn. 2016. Defining coherent vortices objectively from the vorticity. Journal of Fluid Mechanics 795:136-173 https://doi.org/10.1017/jfm.2016.151.

Henry, D. 2013. Dispersion relations for steady periodic water waves with an isolated layer of vorticity at the surface. Nonlinear Analysis: Real World Applications 14:1,034-1,043, https://doi.org/10.1016/j.nonrwa.2012.08.015.

Henry, D. 2016. Equatorially trapped nonlinear water waves in a $\beta$-plane approximation with centripetal forces. Journal of Fluid Mechanics 804, R1, https://doi.org/ 10.1017/jfm.2016.544.

Ionescu-Kruse, D., and C.I. Martin. 2018. Local stability for an exact steady purely azimuthal equatorial flow. Journal of Mathematical Fluid Mechanics 20:27-34, https://doi.org/10.1007/s00021-016-0311-4.
Ko, J., and W. Strauss. 2008. Effect of vorticity on steady water waves. Journal of Fluid Mechanics 608:197-215, https://doi.org/10.1017/S0022112008002371.

Liu, C., A. Köhl, Z. Liu, F. Wang, and D. Stammer. 2016. Deep-reaching thermocline mixing in the equatorial pacific cold tongue. Nature Communications 7, 11576, https://doi.org/10.1038/ncomms11576.

Martin, C.I. 2014. Dispersion relations for rotational gravity water flows having two jumps in the vorticity distribution. Journal of Mathematical Analysis and Applications 418:595-611, https://doi.org/10.1016/j.jmaa.2014.04.014.

Martin, C.I. 2015. Dispersion relations for gravity water flows with two rotational layers. European Journal of Mechanics - B/Fluids 50:9-18, https://doi.org/10.1016/ jeuromechflu.2014.10.005.

Martin, C.I. 2017a. On periodic geophysical water flows with discontinuous vorticity in the equatorial f-plane approximation. Philosophical Transactions of the Royal Society A 376, https://doi.org/10.1098/rsta.2017.0096.

Martin, C.I. 2017b. On the existence of free-surface azimuthal equatorial flows. Applicable Analysis 96:1,207-1,214, https://doi.org/10.1080/00036811.2016. 1180370.

Martin, C.I. 2017c. Two-dimensionality of gravity water flows governed by the equatorial f-plane approximation. Annali di Mathematica Pura ed Applicata 196:2,253-2,260, https://doi.org/10.1007/s10231-017-0663-2.

Martin, C.I., and B.-V. Matioc. 2016. Gravity water flows with discontinuous vorticity and stagnation points. Communications in Mathematical Sciences 14:415-441, https://doi.org/10.4310/CMS.2016.v14.n2.a5.

Milne-Thomson, L.M. 1960. Theoretical Hydrodynamics. The Macmillan Co. New York, 768 pp.

Münchow, A., T.J. Weingartner, and L.W. Cooper. 1999. The summer hydrography and surface circulation of the East Siberian Shelf Sea. Journal of Physical Oceanography 29:2,167-2,182, https://doi.org/10.1175/1520-0485(1999)029 $<2167:$ TSHASC>2.0.CO;2.

Peregrine, D.H., and I.G. Jonsson. 1983. Interaction of Waves and Currents. Report of the U.S. Army Coastal Engineering Research Center 83-6, 88 pp.

Ribeiro, R., P.A. Milewski, and A. Nachbin. 2017. Flow structure beneath rotational water waves with stagnation points. Journal of Fluid Mechanics 812:792-814, https://doi.org/10.1017/jfm.2016.820.

Smyth, W.D., J.N. Moum, and J.D. Nash. 2011. Narrowband oscillations in the upper equatorial ocean. Part II: Properties of shear instabilities. Journal of Physical Oceanography 41:412-428, https://doi.org/10.1175/2010JPO4451.1.

Swan, C., I.P. Cummins, and R.L. James. 2001. An experimental study of two-dimensional surface water waves propagating on depth-varying currents. Journal of Fluid Mechanics 428:273-304, https://doi.org/10.1017/S0022112000002457.

Talley, L.D., G.L. Pickard, W.J. Emery, and J.H. Swift. 2011. Descriptive Physical Oceanography: An Introduction. Academic Press, 560 pp.

Thomas, G.P. 1990. Wave-current interactions: An experimental and numerical study. Journal of Fluid Mechanics 216:505-536, https://doi.org/10.1017/ S0022112090000519.

Toggweiler, J.R., and H. Bjornsson. 2000. Drake Passage and palaeoclimate. Journal of Quaternary Science 15:319-328, https://doi.org/10.1002/10991417(200005)15:4<319::AID-JQS545>3.0.CO;2-C

Umeyama, M. 2012. Eulerian-Lagrangian analysis for particle velocities and trajectories in a pure wave motion using particle image velocimetry. Philosophical Transactions of the Royal Society A 370:1,687-1,702, https://doi.org/10.1098/ rsta.2011.0450.

Vallis, G.K. 2006. Atmospheric and Oceanic Fluid Dynamics. Cambridge University Press, $745 \mathrm{pp}$.

Wahlen, E. 2009. Steady water waves with a critical layer. Journal of Differential Equations 246:2,468-2,483, https://doi.org/10.1016/j.jde.2008.10.005.

Wahlen, E. 2014. Non-existence of three-dimensional travelling water waves with constant non-zero vorticity. Journal of Fluid Mechanics 746, https://doi.org/ 10.1017/jfm.2014.131.

Walton, D.W.H., ed. 2013. Antarctica: Global Science from a Frozen Continent. Cambridge University Press, Cambridge, 352 pp.

\section{ACKNOWLEDGMENTS}

This research was supported by the Science Foundation Ireland grant "Nonlinear wave-current interactions in the nearshore: Addressing the role of vorticity and nonlinearity in the modelling of ocean energy." The author would like to thank the referees and W.D. Smyth for comments and suggestions that improved the paper.

\section{AUTHOR}

Calin Iulian Martin (calin.martin@ucc.ie) is a researcher at the School of Mathematical Sciences, University College Cork, Ireland.

\section{ARTICLE CITATION}

Martin, C.I. 2018. On the vorticity of mesoscale ocean currents.

Oceanography 31(3):28-35, https://doi.org/10.5670/oceanog.2018.306. 